\title{
Small Food Businesses: Trying to Improve Taking Records with a Mobile Device Application
}

\author{
Esteban Pérez, António Raposo, Conrado Carrascosa, Esther Sanjuán, Cristina Mauricio, Rafael \\ Millán \\ University of Las Palmas de Gran Canaria, Las Palmas, Spain \\ E-mail: estebanpg@gmail.com \\ Received March 15, 2011; revised April 12, 2011; accepted April 20, 2011
}

\begin{abstract}
PDA's (Personal Digital Assistant) and mobile phones have become multitasking devices that provide an enormous potential in various fields, including HACCP. We have designed a record taking software in order to be used in small food catering establishments, like canteens or restaurants. It's developed for Windows Mobile ${ }^{\circledR}$ for use in mobile devices such as PDA's and Smartphone's. It enables the creation of four types of records: -Reception; -Temperature; -Cleaning; -Chlorine level in water. It's possible to export data to common file formats like .pdf or .word. An Android OS version is currently under development.
\end{abstract}

Keywords: HACCP, Food Control, Hygiene, New Technologies, Taking Records

\section{Introduction}

In our day to day new technologies are increasingly present, providing, in most cases, the performance of our daily tasks.

The field of hygiene and food safety, in particular to do with the system of Hazard Analysis and Critical Control Points (HACCP), does not remain oblivious to new technologies, but there are still areas where it has not been reached deploy with the force that, in our opinion, should. Thus, although the big players in the big food companies are using computerized systems that allow management of the system, this is not the case of small food establishments (restaurants and cafes).

Data available from the EU indicate that about $90 \%$ of the European Union's food industry is made up of small or medium-sized enterprises [1].

Those numbers show how important this sector is.

In this work, a tool based on use of mobile devices (Smartphone's PDA's) is developed for making records in these small establishments.

\subsection{Food Hygiene and HACCP}

Hygiene is needed in food establishments for the production of healthy food (not harmful to health), and satisfactory from the alimentary (nutrition) and commercial (good conservation, consumer confidence about the overall quality) point of view. To obtain food quality is required the use of healthy raw materials and to respond to a fold of strict conditions, to avoid contamination (limiting, removing or destroying) during processing.

The term "hazard analysis and critical control point (HACCP)" was first introduced in the European Directive 93/43/CE (1993) [2].

The HACCP system identifies critical control points in the production process that are essential to monitor and control product's safety. HACCPs preventive focus is seen as more effective than testing a product and then destroying or reworking it [3].

HACCP is widely recognized in the food industry as a preventive system for managing food safety [4].

In HACCP trust various international organizations such as WHO or FAO, and governments of many countries, including Spain.

Flexibility is an element to take into account in the system, since there are many food activities, and each of them has particular problems and characteristics. In some cases, the adoption of good hygiene practice guides can be used in place of a HACCP system.

In Spain, the HACCP self-control system began to be a legal requirement for food businesses by the RD 2207/ 1995 laying down health rules concerning foodstuffs (which happens to be the transposition of Directive 93 / 43/CEE).

The current rules in Regulation (EC) 852/2004 on the 
Table 1. Data provided by the Spanish Federation of Hoteliers (FEHR) for number of restaurants and cafes in 2007[5].

\begin{tabular}{lll}
\hline & $\mathrm{N}^{\mathrm{o}}$ Restaurants & $\mathrm{N}^{\mathrm{o}}$ Cafes \\
\hline CC.AA.Canarias & 6.816 & 1.626 \\
Spain & 81.989 & 15.624 \\
\hline
\end{tabular}

hygiene of foodstuffs, in Chapter II which refers to the obligations of food business operators, in particular in Article 5 provides that it is HACCP principles to establish a system of documentation concerning all procedures and records appropriate to the principles of HACCP and its implementation.

HACCP is a prospective system based on seven principles [6]:

Principle 1: Conduct a hazard analysis.

Principle 2: Determine the critical control points (CCP's).

Principle 3: Establish critical limit.

Principle 4: Establish a system to monitor control of CCP's.

Principle 5: Establish the corrective action to be taken when monitoring indicates that a particular CCP is not under control.

Principle 6: Establish procedures for verification to confirm that the HACCP system is working effectively.

Principle 7: Establish documentation concerning all procedures and records appropriate to these principles and their application.

This work will be of particular interest to the achievement of the last of these principles.

Record keeping not only focuses the attention of the employees on their roles in achieving food safety but also provides a means to document and verify that correct procedures have been followed [7]. [8]:

The main positive elements of the HACCP system are

- It helps to set priorities.

- Allows for planning how to avoid problems rather than waiting for control to occur. It is, therefore, a preventive system.

-Eliminates the unnecessary use of resources in superfluous considerations, directing attention to directly control the key factors involved in the health and quality throughout the food chain, resulting in more favorable cost/benefit ratio.

Some authors [9] have highlighted some of the barriers that impede the development and implementation of HACCP plans. Thus, negative environmental factors, points to the lack of time as a major barrier, followed by education/training and economic cost (the latter factor has more weight even if it fits in small catering establishments).
Despite the acknowledged contribution of Small and Medium Enterprises (SMEs) to the food industry there is increasing evidence that Hazard Analysis Critical Control Point (HACCP) implementation is limited in this sector, with the burden of implementation perceived as potentially insurmountable [10].

In general, the factors that make a HACCP system fail are those described by [11]:

- The HACCP plan shall become a "paper exercise" and not put into practice.

- The HACCP plan is very complicated. Do not forget that the HACCP plan must match the type of products made and potential risks that may exist.

There is a risk that the HACCP system can be presented as a bureaucratic nightmare [12], losing its practical significance.

The way in which staff understand and generates attitudes toward HACCP based initially on the first introduction they have about him as well, work should be focused on making introductions accessible, relevant and positive.

The bureaucracy should be minimized to ensure food security, and valued as such.

In addition, small establishments have a different profile than the big chains. They need more support and specific advice and an application of HACCP principles that suits their particular case.

Work has been developed taking into account all these precedents.

\subsection{Mobile Computing Devices and HACCP Environment}

Undoubtedly, new technologies are present every day with more force in our lives, enabling us, in most cases, to perform our daily tasks in a more comfortable and effective way. In the professional field, the current trend is that we are not limited to a static place to develop our work, becoming increasingly blurred the concept of "office” as a physical place to work. Rather, it speaks of "mobile office" to refer to the ability to schedule work freely and, above all, freedom of movement.

In the mid-90's Apple launched the first PDA [13].

Initially, the PDA's were used for little more than an agenda to record tasks and manage contacts, and mobile phones on the other hand, to send and receive voice calls and use short text messages (SMS). The PDA is a tool that allows us to be more productive [14], something that the world of the restoration was realized with portable applications that can streamline the entry of commands, and what we intend to contribute to this work.

Is considered that it is interesting to use HACCP software, as an ally in the task of facilitating the implemen- 
tation of the same in small and medium food businesses, as is the case at hand [10].

The implementation of this system has been gradually, avoiding as much as possible so that users can be overwhelmed by something totally new in its way of work, so it is necessary that staff were previously familiarized with the hardware and with the specific software, with our all time support to resolve any questions or suggestions they may arise.

Thus, shortly before having an initial version of the program, consulting the kitchen manager what their level of knowledge of HACCP and on mobile devices and specific software for HACCP.

\section{What is RegItRest?}

RegItRest is an easy to use software developed for taking records in small and medium food industries (canteens, restaurants) in an easy way using mobile devices like PDA's or Smartphone's running Windows Mobile 2003 or later. (An Android version is currently under development). It allows the creation of 4 types of records: reception record, cleaning records, temperature records, and water chlorine level records.

The saved records keep stored into the device or in a memory card, and are available for further reviews.

Also it has an option to look up some basic statistics in the case of temperature and chlorine records. By this way is possible to generate data like maximum and minimum values, and medium values, in a period of time chose by the user using a calendar interface.

If printing is need, an application for PC running Windows XP or later allows to export data to various common informatics file formats (like .pdf or .doc) and print them later. However, RegItRest is not compatible with other softwares in the food business.

The scientific literature related to the HACCP system implementation issue shows how many authors $[15,16]$ have tried to define procedures aimed at simplifying the hazard analysis. These authors recommend the use of specific software systems which make it easier to analyze/compare the different risks which may stem from production processes.

We can consider some precedents in the use of computers to help HACCP.

One of this is HYGRAM (hygiene risk assessment model), that works on Microsoft Excel, its goal is to provide a practical and easy-to-use model that would help in conducting hazard analysis [15].

Other of these precedents that can be mentioned is RECEPMATRA, a PC software centered in exhaustive reception records in military bases [17].

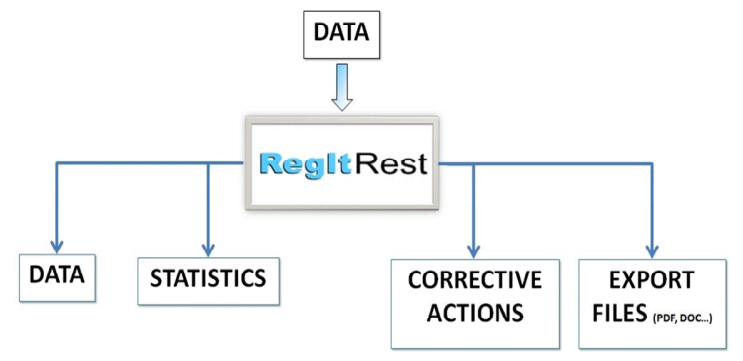

Figure 1. Inputs and Outputs Scheme Application.

\section{RegItRest Benefits}

\section{A) COMFORT}

Data collection and recovery thereof.

It is no longer necessary to make photocopies or have stored large amounts of documentation that in addition to occupying space is subject to mislay.

B) FLEXIBILITY

Multiple compatible devices.

The application is cross platform, compatible with any device equipped mobile operating system Windows Mobile 2003 or later. This allows to choose from a wide variety of devices available in the market and choosing one that is more suited to our needs (autonomy, size, weight...). In addition, it is probably that the user already has one (for personal or professional) of any of these terminals, thus the preliminary adaptation to hardware does not exist.

Later records can be exported to different types of computer files as .doc or .pdf for further sorting and printing if desired.

C) POWER

Storage and processing.

The software works by making maximum use of hardware capabilities and mobile OS, taking into account that these are more limited than those of any PC.

Thus, not only input data are stored, but are also treated to obtain statistical data as mean, minimum and maximum parameters. Also, it generates an automated corrective actions based on the data entered.

D) CONFIDENCE AND IMAGE

Both customers and health authorities.

Clearly, any establishment that has, on one hand a health hygiene advice by qualified veterinary staff and the other a system for recording data, to carry out an objective and continuous verification various processes, with the overall purpose to identify possible weaknesses in the establishment and establish corrective measures to help raise quality standards, it will be a benchmark of reliability and quality by consumers and by the competent authorities health. To any incident, the establishment has a database that the authority may require, effectively prov- 
ing that it takes an effective procedure for making records, thus minimizing hypothetical responsibilities.

The application can work with four types of records:

a) Registration of reception

b) Record temperatures

c) Registration of water chlorination

d) Registry cleaning

a) Registration of Reception

Information collected:

- $\quad$ Element received

- Provider

- Comments

- Possible deficiencies/non-conformities:

Transportation organoleptic characteristics

product temperature

date of minimum durability

b) Record temperatures

Information collected:

- Temperature measured in Celsius degrees, the various equipment at the facility.

c) Record water chlorination

Information collected:

- Level of free residual chlorine, measured in ppm (parts per million) of water within the facility making

d) Cleanout

Information collected:

- $\quad$ Frequency of cleaning equipment (including in this term to floors and walls) of the facility, e.g. daily or shock (the latter performed as often as recommended cleanup plan)

The purpose of this project was to develop a computer program (RegItRest) for making records in catering facilities, which operate on mobile devices with Windows Mobile operating system, such as PDAs (small computers with touch screen) and mobile phones next generation, and also a helper application (ReportRest) aimed at PCs and laptops, which allows automated reporting in different file formats (.doc, .pdf and others) and print them.

RegItRest, from data entered into the system, generates an output comprised of four main elements:

- Data

- Statistics (mean, minimum and maximum)

- Corrective action

- Print reports (using PC-client application)

\section{RegItRest Structure and Technology}

The project consisted of two applications, one in which mobile data is collected and stored in a database and a desktop on which reports are created. Data is synchronized between the two applications and SD memory cards used

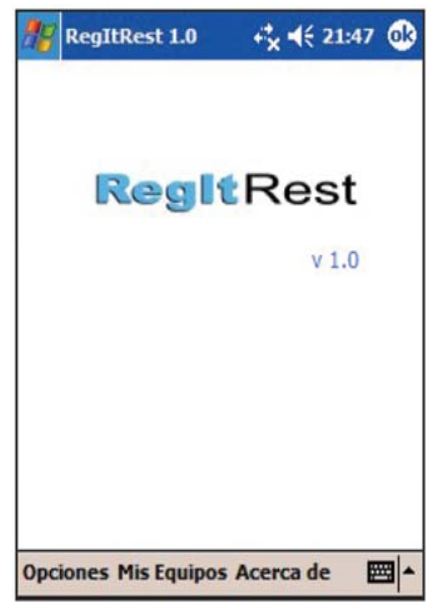

Figure 2. Title Screen.

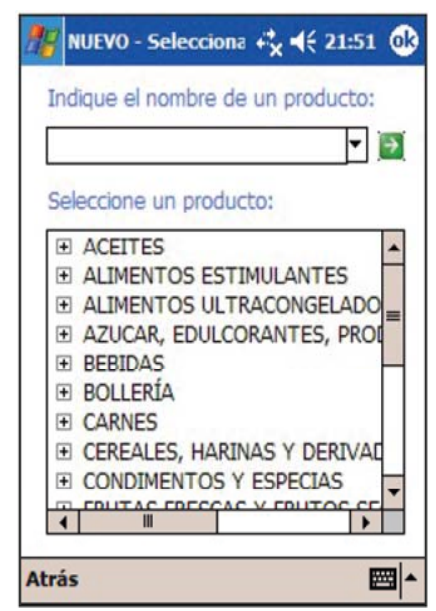

Figure 3. Reception Main Screen.

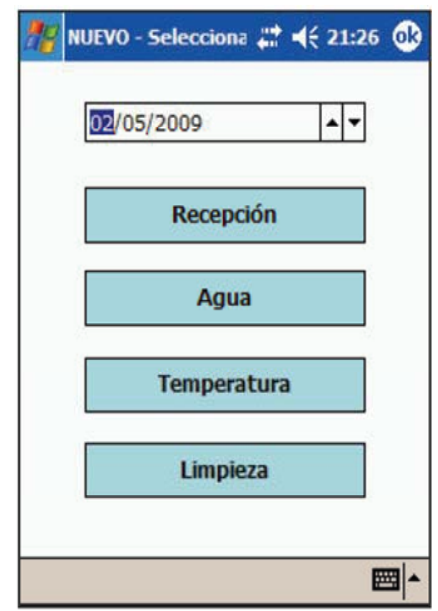

Figure 4. New Record Screen.

for transferring data from the mobile device to the desktop application.

The mobile application development was carried out 
with net Compact Framework using C \# programming language. The application is compatible and can be deployed on mobile devices and Windows Mobile Pocket PC.

The desktop application was programmed using the .net Framework in C \#. It is compatible and can run on any Windows operating system from Windows XP.

The software has been developed using model view controller pattern, which separates business logic from user interface and application data.

As database was used in the mobile version Mobile Edition Microsoft SQL Express, the mobile version of Microsoft SQL. Was done using the SDK provided within the Microsoft suite of tools to develop the layer of abstraction which agreed to the data persisted.

In version was used as the database engine Microsoft SQL Express.

For reports was used for the desktop version of Crystal Reports and the mobile version diagrams were measured using the .net Compact Framework.

It also created an installer to install the program and the libraries necessary to the operation of both the mobile version as on the desktop.

\section{RegItRest Stability, Speed and Efficency}

During the period of three months, this application was tested in four different catering establishments belonging to the University of Las Palmas de Gran Canaria, with great receptivity on the part of users. After the first two weeks learning to handle the program in all institutions there has been an outstanding success, and there is no error and was even mentioned by the users who was notorious for greater speed and safety record and data storage.

\section{ReportRest}

This application works on desktops and laptops running Windows XP and later. Its main utility lies in importing RegItRest files generated by the mobile device and generate automated reports on various computer formats (.doc or .pdf and others) and also allows subsequent printing of them.

\section{Results/Conclusions}

RegItRest makes use of Microsoft technologies .net compact framework and SQL server to create a simple interface to the powerful rather than when working with multiple parameters located in a database.

It is compatible with mobile devices equipped with Windows Mobile 2003 or later, and its optimal resolution of 240x320 pixels.
It was paramount when developing it, serve two purposes: ease of use and practicality.

To achieve the first, special attention has been paid to create a user interface straightforward and intuitive, avoiding unnecessary devices and functions. We must take into account the particularities of mobile devices, especially to have a small size screen (with the consequent difficulty of introducing large amounts of data) and a processing capacity of less than more powerful desktop.

For the latter, we have used our own experiences and from fellow vets in matters pertaining to food hygiene, in addition to reference the current legislative framework and different material, scientific literature, to focus on gathering information strictly necessary to achieve a good tool to meet both the food business operators and their staff, as well as inspection and audit services in health and hygiene, whether they are of private or public.

Thus, we think we have designed a pioneering application in its field, useful both for handling staff and for audit or inspection services, facilitating the task of making records, and retrieval of information from them in the verification process.

In future versions of the software, it will be interesting to implement wireless connectivity, allowing send data to a server, with the objective, in one hand to manage in a better way the information, and in the other hand, to be able to verify in real time the records by the food hygiene responsible.

\section{Acknowledgements}

Part of the development of this application has been financed thanks to a grant provided by "Fundación Universitaria de Las Palmas” and its INNOVA program.

\section{References}

[1] E. Taylor, "HACCP in Small Companies: Benefit or Burden?” Food Control, Vol. 12, No. 4, 2001, pp. 217-222. doi:10.1016/S0956-7135(00)00043-8

[2] “European Council Directive 93/43/EEC, Official,” Journal of the European Communities, No, L 175/2, 19. 7. 93, 1993.

[3] International Commission on Microbiological Specification for Foods, "Application of the Hazard Analysis Critical Control Point System to Ensure Microbiological Safety and Quality”, Blackwell Scientific Publications, Oxford, Vol. 4, 1998.

[4] M. D. Pierson and J. D. A. Corlett, "HACCP: Principles and Applications”, Van Nostrand Reinhold, New York, 1992.

[5] “Spanish Catering Trade Federation”, Los Sectores de La Hostelería en 2007, Madrid. 
[6] "Hazard Analysis and Critical Control Point System and Guidelines for Its Applications”, Codex Alimentarius Commission, Roma, 1997.

[7] M. Setiabuhdi, M. Theis and J. Norback "Integrating Hazard Analysis and Critical Control Point (HACCP) and Sanitation for Verifiable Food Safety", Journal of the American Dietetic Association, Vol. 97, No. 8, 1997, pp. 889-891. doi:10.1016/S0002-8223(97)00217-4

[8] E. Guzman Torres, A. Rodríguez Matos, M. Otero Fernández, O. Moreno Sanchez, "El Análisis de Peligros y Puntos Críticos de Control Como Instrumento Para la Reducción de los Peligros Biológicos”, Revista Electrónica de Veterinaria, Vol. 6, No. 9, 2005.

[9] A. Ramírez Vela and J. Martín Fernández, "Barriers for the Developing and Implementation of HACCP Plans: Results from a Spanish Regional Survey”, Food Control, Vol. 14, No. 5, 2003, pp. 333-337. doi:10.1016/S0956-7135(02)00098-1

[10] Taylor and K. Kane, "Reducing the Burden of HACCP on SMEs”, Food Control, Vol. 16, No. 10, 2005, pp. 833-839. doi:10.1016/j.foodcont.2004.06.025

[11] R. T. Mitchell, "Why HACCP Fails", Food Control, Vol. 9, No. 2-3, 1998, pp. 101-103. doi:10.1016/S0956-7135(98)00084-X

[12] E. Taylor, J. Z. Taylor, "Perceptions of "the Bureaucratic Nightmare of HACCP”, British Food Journal, Vol. 106,
No 1, 2004, pp. 65-72. doi:10.1108/00070700410515217

[13] J. Anton I Riera, J. C. Juárez Giménez, N. Aznar Sorribes, M. Boixadera Vendrell, C. Ibáñez Collado, J. Monterde-Junyent, "Ordenadores de Bolsillo", Revista ROL de Enfermería, Vol. 31-1, 2008, pp. 57-65.

[14] L. C. Larsson, "Improving Your Productivity with a PDA: Some Suggestions”, Library High Technology, Vol. 21, No 4, 2003, pp. 426-439. doi:10.1108/07378830310509736

[15] P. Tuominen, S. Hielm, K. Aarnisalo, L. Raaska and R. Maijala, "Trapping the Food Safety Performance of a Small or Medium-Sized Food Company Using a Risk-Based Model, The HYGRAM ${ }^{\circledR}$ System”, Food Control, Vol. 14, No. 8, 2003, pp. 573-578. doi:10.1016/S0956-7135(02)00147-0

[16] S. J. C. Van Gerwen, J. C. de Wit and M. H. Notermans, "An Identification Procedure for Foodborne Microbial Hazards”, International Journal of Food Microbiology, Vol. 38, No. 1, 1997, pp. 1-15. doi:10.1016/S0168-1605(97)00077-9

[17] R. Millán, “Diseño y Desarrollo de un Sistema Informatizado de Recepción de Alimentos Como Herramienta de Seguridad Alimentaria en el Entorno APPCC”, Seguridad Alimentaria y Sanidad Ambiental, Madrid, 2003. 